\title{
Automated segmentations of skin, soft-tissue, and skeleton from torso CT images
}

\author{
Xiangrong Zhou*a, Takeshi Hara ${ }^{\mathrm{a}}$, Hiroshi Fujita ${ }^{\mathrm{a}}$, Ryujiro Yokoyama ${ }^{\mathrm{b}}$, \\ Takuji Kiryu ${ }^{\mathrm{b}}$, and Hiroaki Hoshi ${ }^{\mathrm{b}}$ \\ ${ }^{a}$ Department of Intelligent Image Information, Division of Regeneration and Advanced Medical \\ Sciences, Graduate School of Medicine, Gifu University, Yanagido 1-1, Gifu 501-1193, Japan \\ ${ }^{\mathrm{b}}$ Department of Radiology, Gifu University School of Medicine \& University Hospital, \\ Tsukasamachi 40, Gifu 500-8705, Japan
}

\begin{abstract}
We have been developing a computer-aided diagnosis (CAD) scheme for automatically recognizing human tissue and organ regions from high-resolution torso CT images. We show some initial results for extracting skin, soft-tissue and skeleton regions. 139 patient cases of torso CT images (male: 92, female: 47, age: 12-88) were used in this study. Each case was imaged with a common protocol $(120 \mathrm{kV} / 320 \mathrm{~mA})$ and covered the whole torso with 0.63 (mm) isotopic spatial resolution and 12 (bits) density resolution. A gray-level thresholding based procedure was applied to separate the human body from background. The density and distance features to body surface were used to determine skin, and separate soft-tissue from the other regions. A 3-D region growing based method was used to extract skeleton. We applied this system to 139 cases and found that the skin, soft-tissue and skeleton regions were recognized correctly for $93 \%$ patient cases. The accuracy of segmentation results was acceptable by evaluating the results slice by slice. This scheme will be included in a CAD system for detecting and diagnosing the abnormal lesions in multi-slice torso CT images.
\end{abstract}

Keywords: Mult-slice torso CT images, 3-D image processing, organ region segmentations, CAD

\section{INTRODUCTION}

The newest multi-detector CT (MDCT) scanners have the capacity to scan whole human torso region within one-time CT photography in a high image quality (about $0.63(\mathrm{~mm})$ spatial resolution in 3-D). This not only provides the ability to radiologists to view details of all internal organ regions at same time, but also gives a possibility to analyze the human structure that was presented by different organs and their relationships, which is one of the most important references for abnormality detection. However, high-resolution torso CT images have almost 1000 slices of CT images for each patient case. It is impossible for a radiologist to interpret all the slices manually in front of a monitor screen (or film) without any assistance. In order to reduce the burden of CT image interpretations, recently, the development of computer aided diagnosis (CAD) system is strongly expected.

The request for a CAD system is defined to provide a secondary opinion for a doctor at current stage. That means a CAD system should help radiologists to understand human structure and concentrate attention on suspicious lesions in a wide range of human body. In order to realize such functions, a stable extraction and classification of different organ regions from torso CT images is always necessary and required as the first processing step by a CAD system. Although a lot of research works had been reported for segmenting a special organ region from CT image[1-7], few of them centers on classifying different organ and tissue regions simultaneously and recognizing human torso structure from high-resolution CT images.

We have been developing a CAD system that can recognize normal human structures by segmenting 9 kinds of organ and tissue regions automatically from multi-slice chest CT images. We applied this system to recognize the lung structure from 3 patient cases of multi-slice chest CT images and confirmed the effectiveness and usability of our system from preliminary results[8-10]. This time, we expand this system for recognizing the human tissue and organ regions from torso CT images and use 139 patient cases of high-resolution multi-slice torso CT images to evaluate the performance of our system. In this paper, we firstly describe the methods in this system for automated identifying of skin, soft-tissue(fat) and skeleton regions. And then, we show the extraction results from real CT images and give a conclusion finally. 


\section{METHOD}

The outline of our processing flow is shown in Fig.1. From the density histogram of torso CT images, we discovered that CT image could be divided into 4 regions (air, fat, muscle\&organs, skeleton) roughly by a thresholding method based on density feature. However, Skeleton structure cannot be extract completely only based on gray-scale thresholding in most cases, we add the feature of connectivity in each voxel of CT image to refine the skeleton regions. For skin and fat region classification, we add the feature of 3-D distance from body surface to assist the skin region identification. We show the details of our method in following sessions.

\subsection{Initial region classification from torso CT images}

The purpose of this step is to separate human torso region from background and make an initial classification to divide human body region into 4 parts: fat, muscle (include organ, vessels and et al.), skeleton and air regions. A gaylevel thresholding method is used to identify each above region based on their density distribution. Instead of using fixed threshold values to segment target regions, we provides a histogram analysis method[11] that can be used to decide the optimal threshold value just for the current CT image automatically. The details are shown as following:

Step 1 Preprocessing of CT image: We extract the CT image data slice by slice from DICOM format files and arrange them into a 3-D data array based on the information inside the DICOM heads. This 3-D data array is regarded as the original CT image(Fig.1(a)) that has almost the same spatial resolutions in 3-D.

Step 2 Threshold value selections: This step is to decide the optimal threshold value for segmenting human tissues from the background that is almost air region. We assume that the total region of a CT image just involves 2 parts: air regions and human tissue regions with the different density distributions. We measure the separation degree between those two density distributions on gray level histogram using a discriminate analysis[11] and select the gray level $T_{1}$ that has the maximum separation degree as the optimal threshold value for human tissue segmentation. Due to relation of density distributions in different human tissues, the fat tissue can be separated from the other tissues using CT value of water that is bout $0(\mathrm{HU})$. We use a process to select the best gray level $\mathrm{T}_{2}$ by search the zero-cross point of differential value from histogram nearest to $0(\mathrm{HU})$ and use it for identifying fat regions. For the voxel that has a CT value larger than $\mathrm{T}_{2}$, we use discriminate analysis again to decide a gray level $\mathrm{T}_{3}$ for separating skeleton and muscle regions roughly.

Step 3 Gary level thresholding: Using the selected threshold value $T_{1}, T_{2}$, and $T_{3}$, we separate human tissues from background as follows:

$$
\begin{aligned}
& \text { Voxel }=>\text { Air }\left(\text { gray level }<=\mathrm{T}_{1}\right) \\
& \text { Voxel } \Rightarrow \text { Fat }\left(\mathrm{T}_{1}<\text { gray level }<\mathrm{T}_{2}\right) \\
& \text { Voxel } \Rightarrow>\text { Muscle\&Organ }\left(\mathrm{T}_{2}<\text { gray level }<\mathrm{T}_{3}\right) \\
& \text { Voxel }=>\text { Initial skeleton }\left(\text { gray level }=>\mathrm{T}_{3}\right)
\end{aligned}
$$

Step 4 Connected component processing: We use a 3-D connectivity analysis to select the connected component with the biggest volume from human tissue regions (gray level $\Rightarrow \mathrm{T}_{1}$ ) as the real torso region Fig.1(b) and delete the rest regions.

Step 5 Surface detecting and region filling: We extract the outline of human torso using 2-D border following method that tracing the external borderline between human tissue region and air region slice by slice. Then, we simply fill the internal region of the border line and regard it as the torso region (Fig.1(c)).

Step 6 Distance image generation: We generate a distance image (Fig.1(d)) from the body surface of human torso as the preparation of the next processing step using 3-D Euclidean distance transformation method[12].

\subsection{Skeleton region extraction}

We refine the initial skeleton region (Fig.1(e)) by a 3-D region growing method. Because the extraction accuracy using 3-D region growing is very sensitive to the threshold value that used for controlling the region expansion, we proposed a method to find out the optimal threshold. We set the initial skeleton region extracted in previous step as the "seed points" and really expand the initial skeleton region gradually by increasing the threshold value until the skeleton 




(f)
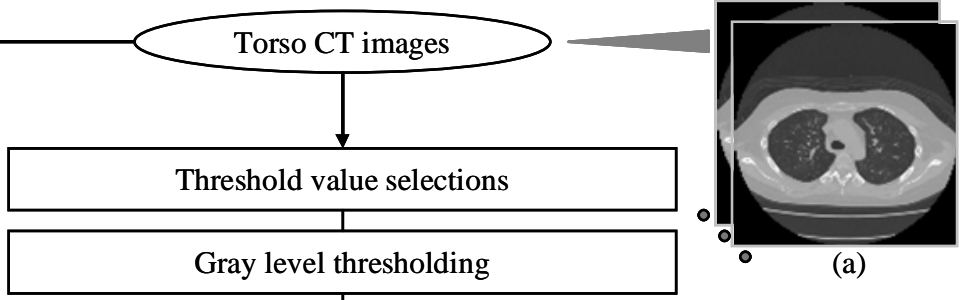

(a)

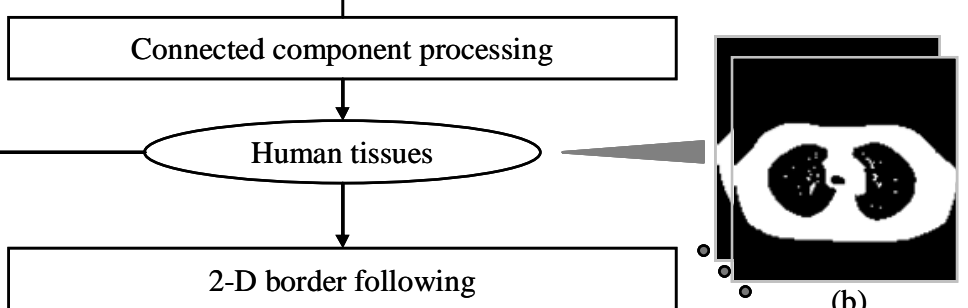

(b)

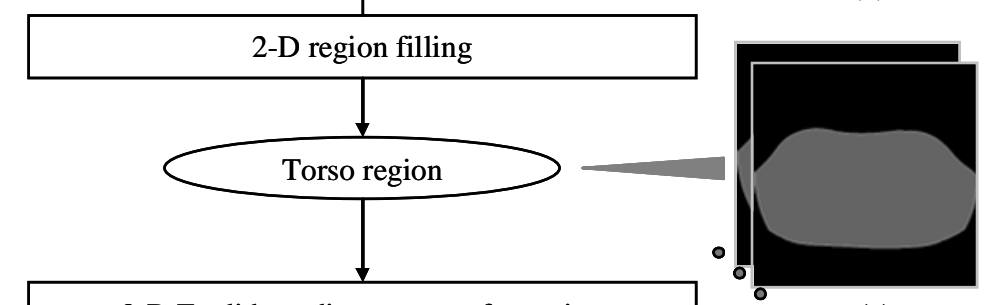

(c)

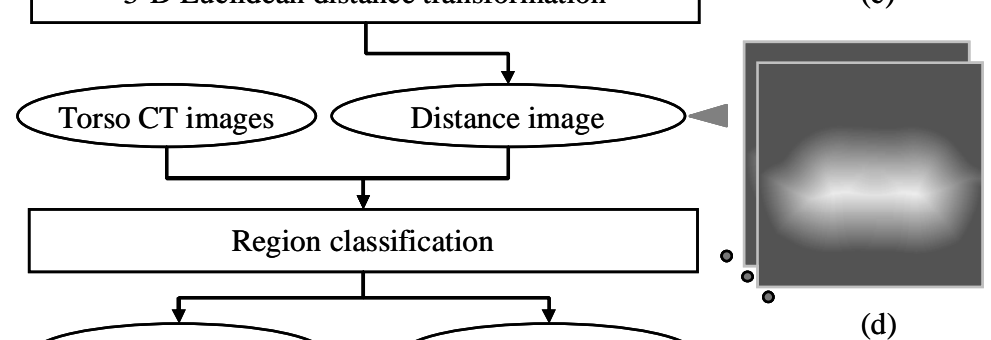

(d)

Fig. 1. Processing flow of skin, fat and skeleton extractions from torso CT images.

region leaks into muscle regions and regard the threshold value just prior to the region leak as the optimal one. The judgment for region leak is simply by watch whether or not the volume increase of the segmented skeleton region goes beyond a limitation during the repetition of the 3-D region growing process. Finally, we output the result of region growing process using the optimal threshold value as the final skeleton region (Fig.1(f)).

\subsection{Skin and fat region identifications}

The skin covers on human body surface with about $2(\mathrm{~mm})$ thickness and present as a high density region in CT images. Here, we identify the skin region using two features (CT value and distance from body surface). We regard the 
voxel whose distance from body surface is less than $5(\mathrm{~mm})$ as the candidacy skin region. Within candidacy skin region, we separate skin from fat regions by a thresholding process based on density distribution and refine the final skin region (Fig.1(h)) using a connected component processing method. The density of fat tissue is always below 0 HU, so that we can easily get it (Fig.1(g)) using the gray-level thresholding from original CT images.

\section{EXPERIMENTS AND RESULTS}

We evaluated the performance of our method using 139 patient cases (male: 92, female: 47) of torso multi-slice CT images. The age of the patients was ranged from 12 to 88 years old. All of those CT images were generated by a multislice CT scanner (LightSpeed Ultra of GE Medical System) using a common protocol (120kV/320mA). After DICOM format decoding, each CT image was expressed as a $512 * 512 * 800-1200$ matrix that covers the entire human torso region with the isotropic spatial resolution about $0.63-0.74(\mathrm{~mm})$ and 12 (bit) density resolution. No contrast media had been used during the CT scans.

We developed a special user interface for accuracy evaluation[8]. This interface can show segmentation regions semitransparently on original CT image for each patient case. The human expert can use this tool to give an evaluation intuitively and quickly by viewing the segmentation results comparing with original CT images from 3-direction sliceby-slice and 3-D shape of segmentation regions. In our experiments, we used this interface to evaluate the accuracy of automatic extraction results of skin, fat and skeleton.

A part of extraction results is shown in Fig. 2 (a male of 30 years old) and Fig.3 (a female of 38 years old). The results of each patient case were shown in 3-D using a volume rendering techniques from front and backside of human body. We shown the surface of skeleton in Fig.2(c) and Fig.3(c). The skin regions were shown by shape of body surface in Fig.2(a) and Fig.3(a). The fat regions were shown by the shape of muscle surface (inner surface of fat) in Fig.2(b) and Fig.3(b). The computing time for automated extracting skin, fat and skeleton is about 10 minutes per patient case using a personal computer (CPU: P4 3.0 GHz).

\section{DISCUSSIONS}

We confirmed that our method could extract the skin region correctly from 98\% (137/139) patient cases. In 2 patient cases, some infusion tubes that pass through the human body surface from the outside of human body have been misclassified as a part of skin region by our method. For fat region extractions, we confirmed that out method could provide good extractions from all the patient cases. For skeleton region extraction, our method can output a stratified result of skeleton structure from 93\% (130/139) patient cases. Our method was failure to extract skeleton from 9 patient cases due to the artifacts caused by the metals near to spinal columns on CT images.

Further more, we verified the density thresholding values $T_{1}$ and $T_{2}$ that were decided automatically for extracting human surface and fat region from different patient cases and confirmed that the selected threshold value was the optimum one for each patient case. From the above results, we considered that our thresholding value selection method is necessary and effective for adapting the difference of inputted CT images.

\section{CONCLUSIONS}

We are developing a CAD system to recognize human structure automatically and showed segmentation results of skin, fat, muscle from high-resolution torso CT images. We have applied this system to 139 cases and confirmed that our system can output satisfied results of skin, fat and skeleton regions from 130 cases correctly. We are improving our system to recognize other kinds of organ regions such as liver, kidney, spleen and will evaluate the performance of this system with more CT images in the future.

\section{ACKNOWLEDGEMENTS}

Authors thank the members of Fujita's Laboratory and Virtual System Laboratory (VSL) of Gifu University for their collaboration. This research was supported in part by research grants from the Collaborative Centre for Academy/Industry/Government and VSL of Gifu University, in part by the Ministry of Health, Labour, and Welfare under a Grant-In-Aid for Cancer Research and in part by the Ministry of Education, Culture, Sports, Science and Technology under a Grant-In-Aid for Scientific Research, Japanese Government. 


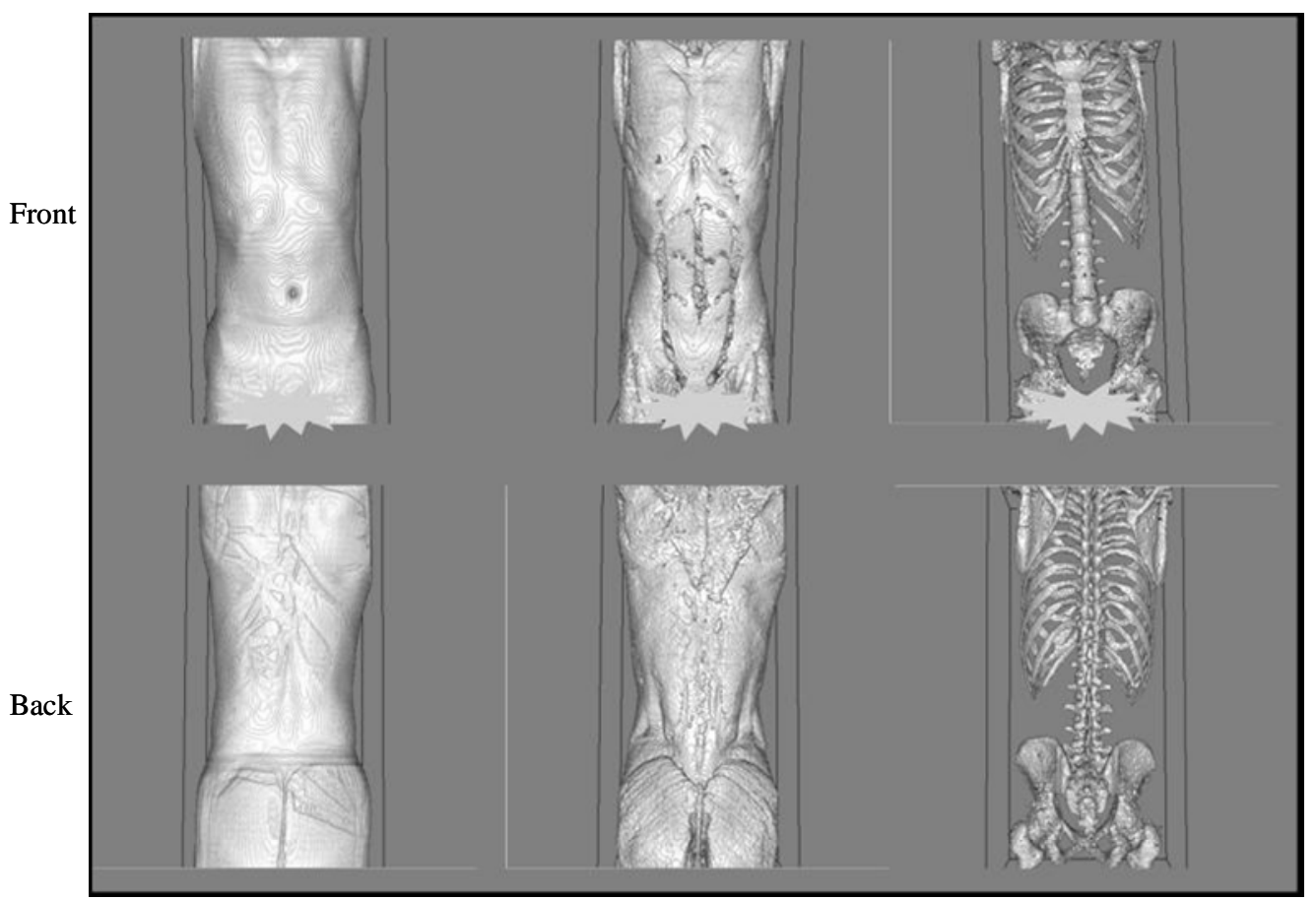

$\begin{array}{lll}\text { (a) Surface of skin } & \text { (b) Surface of muscle after fat deletion } & \text { (c) Surface of skeleton }\end{array}$

Fig. 2. Results of skin, fat and skeleton regions extracted from a case (a male, 30 years old).

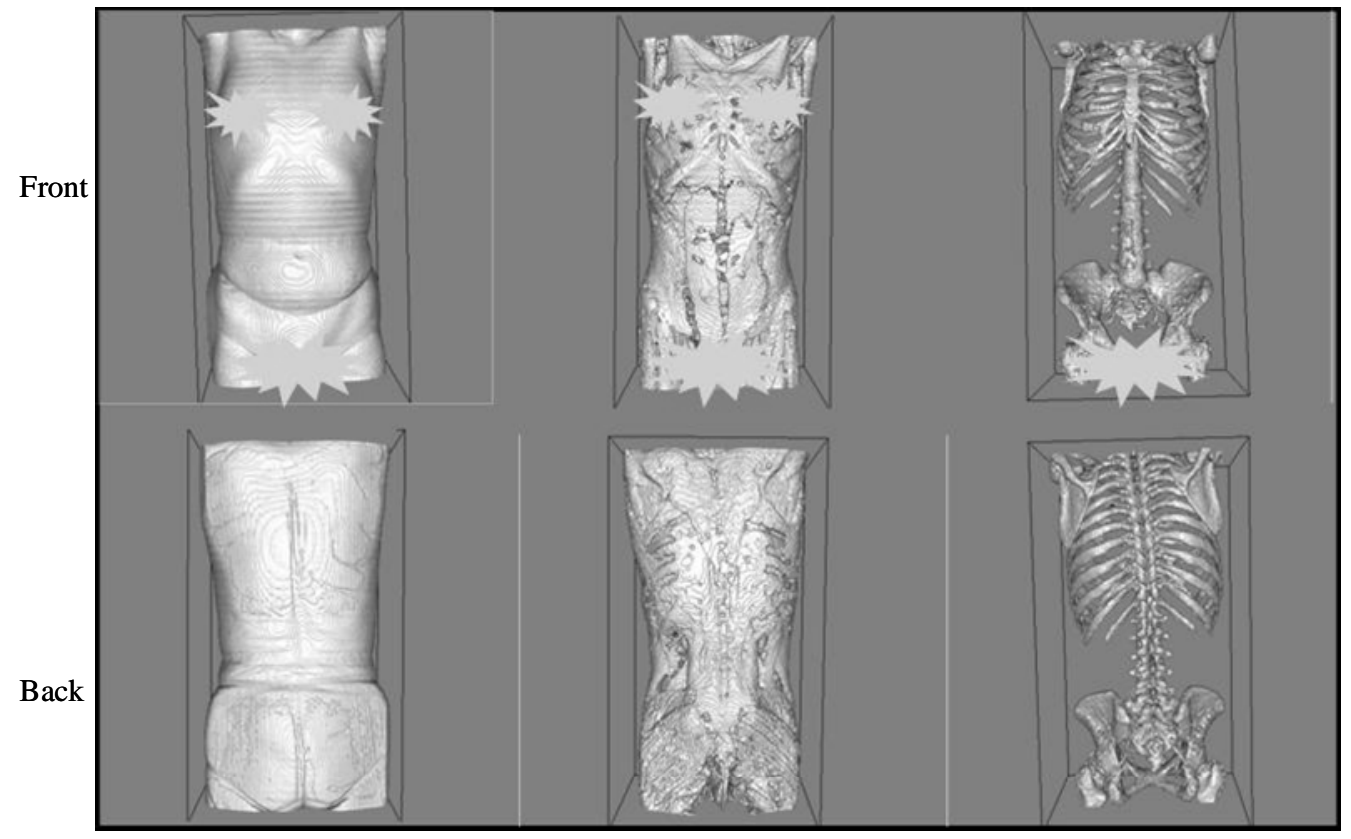

$\begin{array}{lll}\text { (a) Surface of skin } & \text { (b) Surface of muscle after fat deletion } & \text { (c) Surface of skeleton }\end{array}$

Fig. 3. Results of skin, fat and skeleton regions extracted from a case (a female, 38 years old). 


\section{REFERENCES}

1. D. Aykac, E. A.Hoffman, G. Mclennan, and J. M. Reinhardt, "Segmentation and analysis of the human airway tree from three-dimensional X-ray CT images", IEEE Trans. Med. Imag., vol.22, no. 8, pp.940-950, 2003.

2. K. Mori, J. Hasegawa, Y. Suenaga, and J. Toriwaki, "Automated anatomical labeling of the bronchial branch and its application to the virtual bronchoscopy system”, IEEE Trans. Med. Imag., vol. 19, no. 2, pp.103-114, 2000.

3. K. Mori, J. Hasegawa, J. Toriwaki, H.Anno, and K Katada, "Automated extraction of bronchus area from three dimensional X-ray CT images”, IEICE Tech.Rep., vol. 142, 1994. (In Japanese)

4. S. Hu, E. A. Hoffman and J. M. Reinhardt, "Automatic lung segmentation for accurate quantitation of volumetric X-ray CT images”, IEEE Trans. Med. Imag.,vol.20, no. 6, pp.490-498, 2001.

5. J. K., B. Zheng, R. M. Rogers, F. C. Sciurba, Andrew Perez, Brian E. Chapman, Sanjay Patel, Carl R. Fuhrman and David Gur, "Automated lung segmentation in X-ray computed tomography”, Acad. Radiol., vol.10, no. 11, pp.1224-1236, 2003.

6. T.Kitasaka, K.Mori, J.Hasegawa, and J.Toriwaki, “Automated extraction of the lung area from 3-D chest X-ray CT images based upon the 3-D shape model deformation", Proc. of the 13th International Congress and Exhibition on Computer Aided Radiology and Surgery, pp.194-198, 1999.

7. T. Kitasaka, K. Mori, J. Hasegawa, J. Toriwaki and K. Katada, "A method for automated extraction of aorta and pulmonary artery using line models from 3-D chest X-ray CT images with contrast medium”, Proc. of 16th Conf. on Pattern Recognition, pp. III-273-276, 2002.

8. X. Zhou, T. Hara, H. Fujita, Y. Ida, K. Katada, and K. Matsumoto, "Extraction and recognition of the thoracic organs based on 3D CT images and its application", Proc. of the 16th International Congress and Exhibition of Computer Assisted Radiology and Surgery 2002, pp.776-781, 2002.

9. X. Zhou, S. Kobayashi, T. Hayashi, N. Murata, T. Hara, H. Fujita, R. Yokoyama, T. Kiryu, H. Hoshi, and M. Sato, "Lung Structure Recognition: A further study of thoracic organ recognitions based on CT images", Proc. of the 17th International Congress and Exhibition of Computer Assisted Radiology and Surgery 2003, pp.1025-1030, 2003.

10. H. Fujita, X. Zhou, T. Hara, T. Kiryu, R. Yokoyama, and H. Hoshi, “A virtual interpretation training system for chest radiograms using high-resolution multi-slice CT images", Proc. of the 9th International Conference on Virtual Systems and Multimedia, pp.622-628, 2003.

11. N. Otsu, "A threshold selection method from gray-level histogram", IEEE Trans. SMC, vol. SMC-9, no. 1, pp.6266, 1979.

12. T. Saito, and J. Toriwaki, "Euclidean distance transformation for three dimensional digital images", Trans. IEICE, vol. J76-D-II, no.3, pp.445-453, 1993.(In Japanese) 